\title{
Left atrial invasion of a lung cancer: a case report
}

\author{
Fulvio Cacciapuoti ${ }^{1}$, Erica Vetrano ${ }^{2}$, Federico Cacciapuoti ${ }^{3}$ \\ ${ }^{1}$ Cardiology Department, “V. Monaldi” Hospital, Naples; ${ }^{2}$ Post-graduate School of Internal Medicine, "L. Vanvitelli” \\ University, Naples; ${ }^{3}$ Chair of Internal Medicine, "L. Vanvitelli" University, Naples, Italy
}

\begin{abstract}
Lung cancer is the leading neoplastic form worldwide for both incidence and mortality representing the largest contributor to new cancer diagnosis. Cardiac extensions of a pulmonary neoplasm are rare and dramatically under-diagnosed because of the extreme
\end{abstract}

Correspondence: Fulvio Cacciapuoti, MD, Cardiology Department, "V. Monaldi" Hospital, Via L. Bianchi s.n.c., 80100 Naples, Italy.

E-mail: fulvio.cacciapuoti@ospedalideicolli.it

Key words: pulmonary neoplasm; cardiac extension of lung malignancy; hypercalcemia; echocardiography.

Contributions: All the authors made a substantive intellectual contribution, read and approved the final version of the manuscript and agreed to be accountable for all aspects of the work.

Conflict of interest: The authors declare that they have no competing interests, and all authors confirm accuracy.

Availability of data and materials: All data underlying the findings are fully available.

Ethics approval and consent to participate: No ethical committee approval was required for this case report by the Department, because this article does not contain any studies with human participants or animals. Informed consent was obtained from the patient included in this study.

Consent for publication: The patient gave his written consent to use his personal data for the publication of this case report and any accompanying images.

Received for publication: 25 September 2021.

Accepted for publication: 2 November 2021.

Publisher's note: All claims expressed in this article are solely those of the authors and do not necessarily represent those of their affiliated organizations, or those of the publisher, the editors and the reviewers. Any product that may be evaluated in this article or claim that may be made by its manufacturer is not guaranteed or endorsed by the publisher.

${ }^{\text {CC}}$ Copyright: the Author(s), 2021

Licensee PAGEPress, Italy

Monaldi Archives for Chest Disease 2022; 92:2098

doi: 10.4081/monaldi.2021.2098

This article is distributed under the terms of the Creative Commons Attribution-NonCommercial International License (CC BY-NC 4.0) which permits any noncommercial use, distribution, and reproduction in any medium, provided the original author(s) and source are credited. variability of clinical presentation and frequently are expression of an advanced-stage primary lung cancer. The invasion often happens through pulmonary veins in absence of a clear respiratory impairment. Symptoms related to the cardiac involvement as the first presentation of a malignant pulmonary neoplasm are very uncommon and related with poor outcome. Here we present a case of invasion of the left atrium of a pulmonary neoplasm with initial cardiac manifestations and a laboratory finding of hypercalcemia.

\section{Introduction}

Left atrial invasion of a pulmonary cancer, happening via pulmonary veins, is a non-common extension of the respiratory neoplasm [1]. This invasion can be considered a life-threatening event, expression of an advanced-stage disease and can potentially be responsible for several complications such as obstructed pulmonary veins' flows, atrio-ventricular block, atrial fibrillation, ventricular arrhythmias, and others [2]. Lung malignancies are also often responsible for hypercalcemia as an expression of paraneoplastic syndromes and because of bone metastases [3].

\section{Case Report}

A 48-year-old man was taken to our Emergency Department because of sudden onset of palpitations and dyspnea at rest. He also reported progressively worsening cough and hypo-asthenia at lower limbs. No history of cardiovascular risk factors was referred except for a smoke-custom of nearly 20 cigarettes/day. He also referred a weight loss of about $10 \mathrm{kgs}$ in the last year. Tachycardia and systolic murmur were evidenced at cardiac examination. Pulmonary auscultation revealed a slightly diminished vesicular murmur at left hemithorax. The 12-leads EKG shown a paroxysmal supraventricular tachycardia ( 150 beats/min). Blood pressure was $115 / 70 \mathrm{mmHg}$, and $\mathrm{SpO}_{2}$ was $92 \%$. The carotid sinus massage, performed in attempt to correct the supraventricular tachycardia, resulted ineffective but e.v. injection of $10 \mathrm{mg}$ of Verapamil diluted in $250 \mathrm{cc}$ of physiological solution was able to rapidly restore sinus rhythm. Afterwards, the repeated 12 leads EKG showed a sinus rhythm at 90 beats/min, RBB and J-wave in the right precordial derivations (Figure 1). The EKG finding of an "Osborn wave", except for hypothermia, is likely due to an increase of $\mathrm{Ca}^{++}$serum levels [4]. Additional EKG abnormalities in hypercalcemia also include shortened Q-T interval and biphasic $\mathrm{T}$ wave in the right derivations. In this case, the $\mathrm{Ca}^{++}$concentration was of $2.13 \mathrm{mmol} / \mathrm{L}$. 2D-Echocardiography showed values of systolic and diastolic LV dimensions in the normal range. Right ventricle was not dilated, and a valvular tricuspid insufficiency 


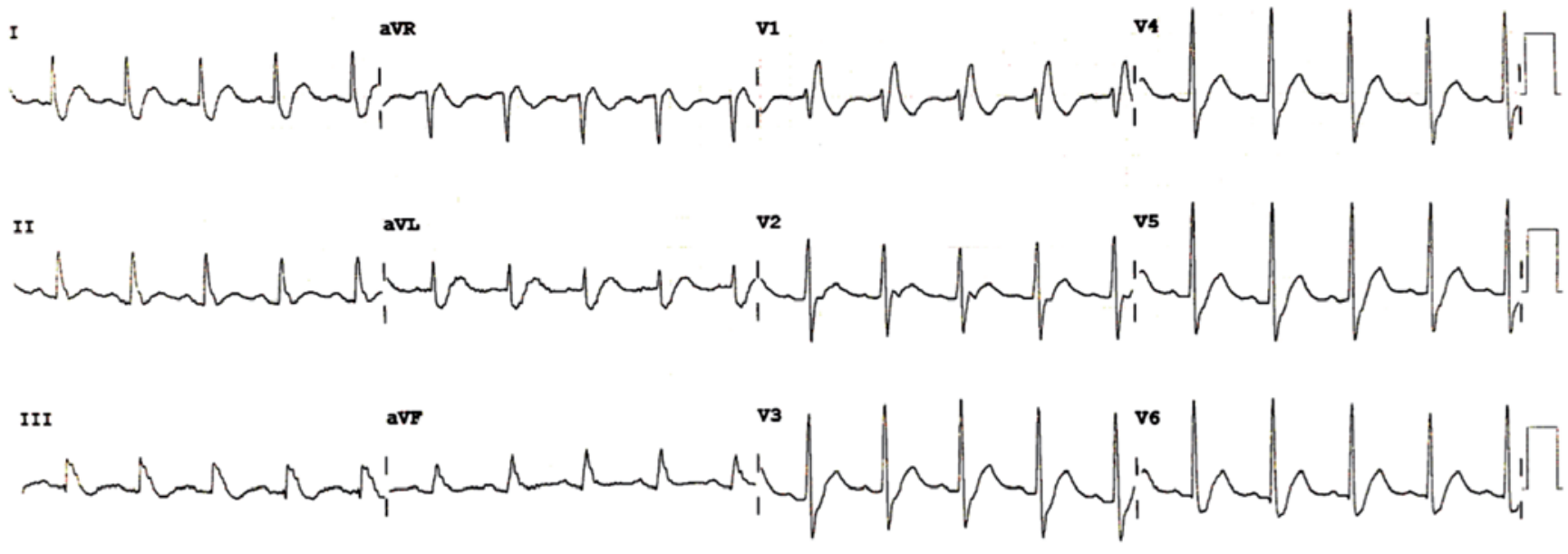

Figure 1. 12 leads EKG-Sinus rhythm 90 beats/min. Right bundle branch block (V1). Reduced Q-T interval evident in the precordial derivations. Biphasic $T$ wave $\left(V_{4}, V_{5}, V_{6}\right)$ and "Osborn" wave $\left(V_{2}\right)$.

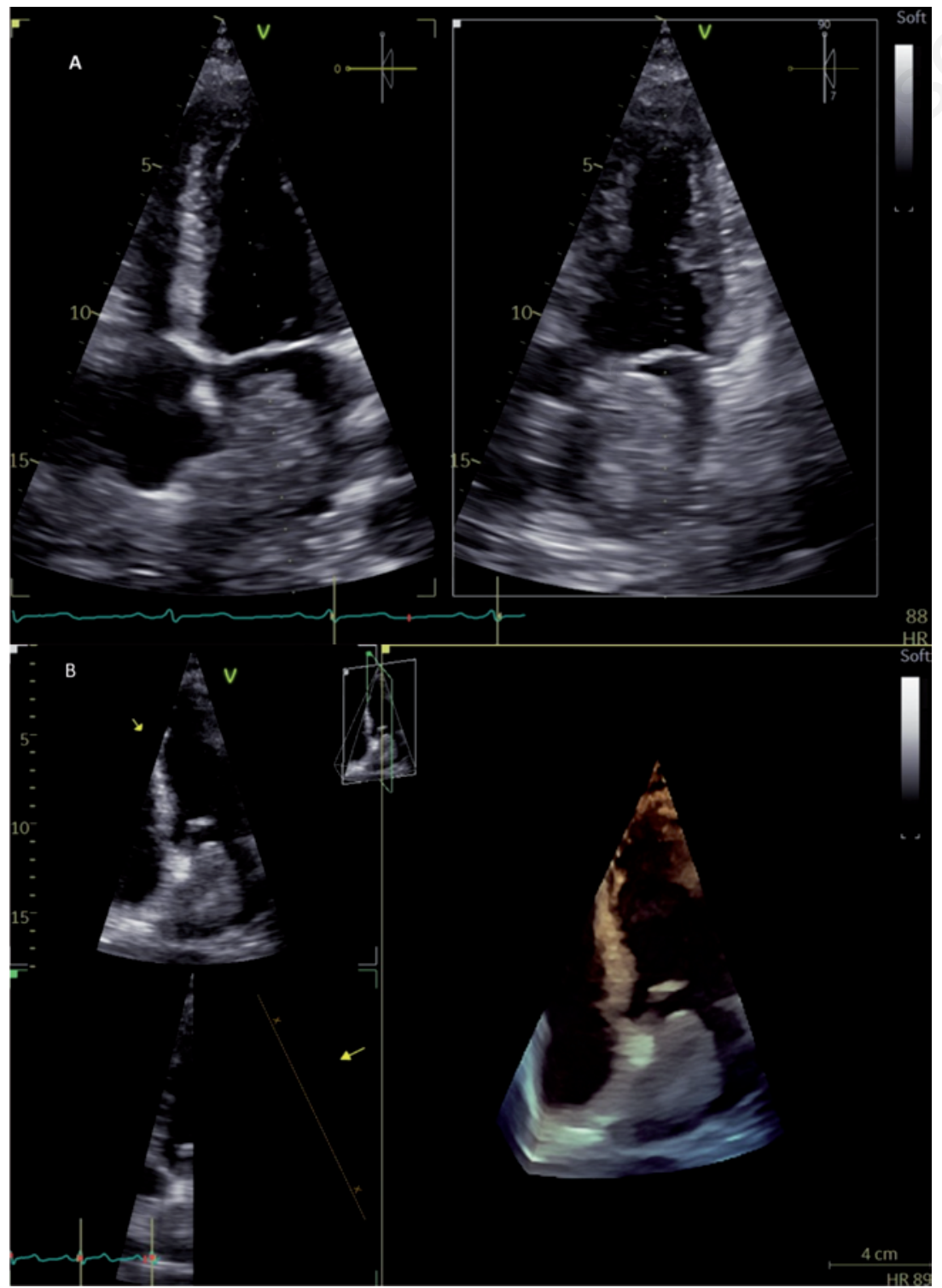

Figure 2. A) 2D transthoracic echocardiography; apical 4 chambers (left) and 2 chambers (right). B) 3D trasnsthoracic echocardiograpy; mass occupying the left atrium. 
was found. In addition, lateral wall of LV was hypokinetic and LVEF\%, evaluated with the Simpson method, was of 50\%. Finally, left atrium revealed a large mass occupying almost completely this chamber. The mass pressed atrial septum, in the absence of an evident inter-atrial shunt (Figure 2A). Three-dimensional echocardiography confirmed the left atrial invasion of the mass (Figure 2B) and a trans-mitral pattern of diastolic disfunction was recorded (Figure 3). Chest radiography showed a mass largely occupying the inferior lobe of left lung, indissociable from the pulmonary tissue. CT scan confirmed the pulmonary image and evidenced its extension in the left atrium through the pulmonary vein occupying it nearly completely (Figure 4). Head CT scan did not show any metastases. On the contrary, multiple metastases were found at whole-body bone scintigraphy.

The morphological and microscopic findings demonstrated that the neoplasm was an adenocarcinoma and it was managed with a combination of surgery and adjuvant therapy performed for

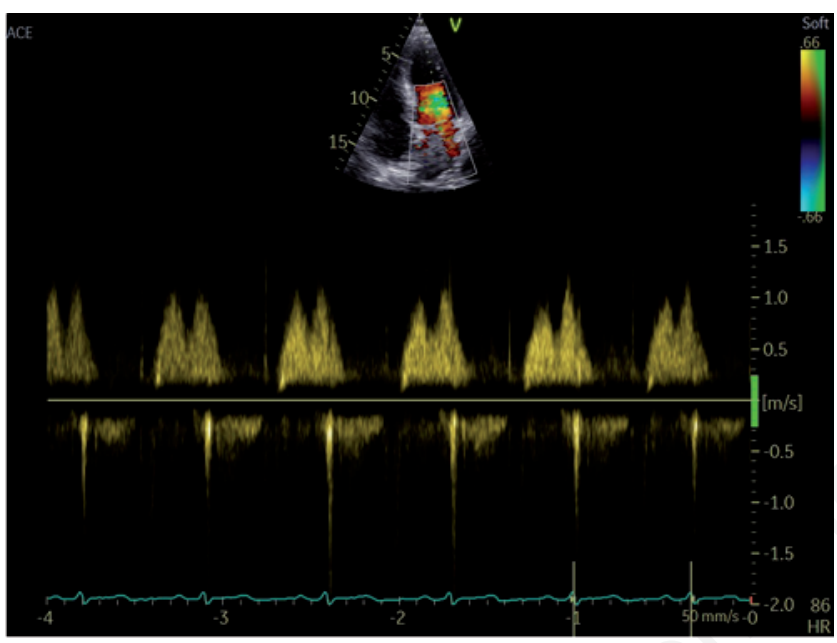

Figure 3. Trans-mitral continuous-wave-Doppler showing the diastolic dysfunction.

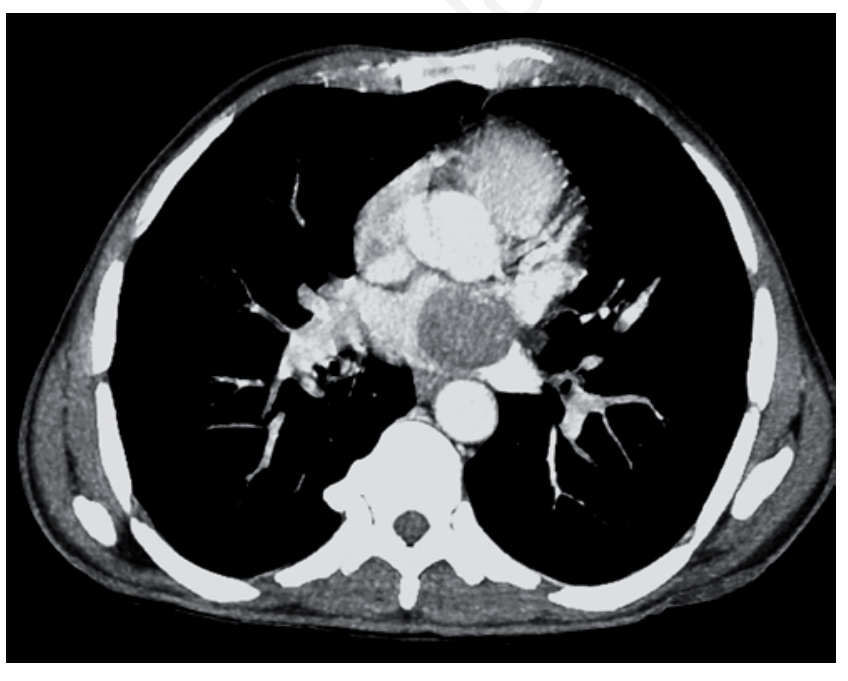

Figure 4. Chest CT scan. Pulmonary neoplasm extended in the left atrium through the pulmonary vein. pulmonary neoplasm, the intracardiac tumoral mass and pulmonary vein tumor. On histopathologic examination, the tumoral mass resulted composed of large pleomorphic cells with ovoid nucleoli. But, three months after surgery, brain TC-scan evidenced multiple brain metastases. The clinical conditions progressively worsened, and the patient died 8 months after surgical treatment.

\section{Discussion}

Left atrial extension of a lung tumor via pulmonary veins is a non-frequent event mimicking left atrial myxoma, firstly showing with cardiac symptoms $[5,6]$. Echocardiography is a method to noninvasively detect the extension of lung neoplasm in the left atrium. Apart from the numerous cardiac complications, the patients with extension of lung cancer into left atrium may have some other organs impairments, such as ischemic stroke $[7,8]$. In the reported case, the laboratory finding of hypercalcemia was a cause of suspect the cancer [9]. Several mechanisms can be responsible for hypercalcemia in malignancy, including parathyroid hormone-related peptide and osteolytic metastases-related hypercalcemia [10]. It's well known, in fact, that Parathyroid Hormone-related Protein (PTHrP) can be responsible for humoral hypercalcemia of malignancies (HHM) through endocrine action and tumor-induced bone destruction [11]. It must be also added that, in our patient, the multi-parametric evaluation (clinical symptoms, physical examination, EKG, echocardiography, chest X-ray, CT scan and laboratory findings) allowed to suspect and recognize the pulmonary malignancy and its left atrial extension $[12,13]$.

\section{References}

1. Lin MT, Ku SC, Wu MZ, Yu CJ. Intracardiac extension of lung cancer via the pulmonary vein. Thorax 2008;63:1122.

2. Weinberg BA, Conces DJ Jr, Waller BF. Cardiac manifestations of noncardiac tumors. Part I: Direct effects. Clin Cardiol 1989;12:289-96.

3. Stewart AF. Clinical practice. Hypercalcemia associated with cancer. N Engl J Med 2005;352:373-9.

4. Otero J, Lenihan DJ. The "normothermic" Osborn wave induced by severe hypercalcemia. Tex Heart Inst J 2000;27:316-7.

5. Cipriano F, Dessoti LU, Rodrigues AJ, et al. Report of a lung carcinoma extended to the left atrium through pulmonary vein. J Thorac Dis 2018;10:E46-E51.

6. Watanabe N, Kubo K. Images in cardiology: Intra-left atrial invasive mass extended via the pulmonary vein. Heart 2001;85:271.

7. Ballo P, Laureano R, Briganti M, et al. Left atrial mass invasion from pulmonary neoplasm extension via the right upper pulmonary vein presenting as ipsilateral stroke. Case Rep Med 2016;2016:7084234.

8. Brandt RR, Rubin J, Reeder GS. Intracardiac extension of a lung tumor causing left ventricular inflow obstruction. J Am Soc Echocardiogr 1995;8:930-3.

9. Chandra R, Abugroun A, Goldberg A, et al. Small cell lung cancer invading the left atrium with subsequent malignant embolic stroke: A case report and review of literature. Cardiol Res 2019;10:188-92.

10. Hamilton F, Carroll R, Hamilton W, Salisbury C. The risk of 
cancer in primary care patients with hypercalcaemia: a cohort study using electronic records. Br J Cancer 2014;111:1410-2.

11. Edwards CM, Johnson RW. From good to bad: The opposing effects of PTHrP on tumor growth, dormancy, and metastasis throughout cancer progression. Front Oncol 2021;11:644303.
12. Mirrakhimov AE. Hypercalcemia of malignancy: An update on pathogenesis and management. N Am J Med Sci 2015;7:483-93.

13. Htwe KK, Aung TT. Diagnostic challenges of a mass in the left atrium. Cureus 2020;12:e8620. 